\title{
Index
}

ADITYA, 242

AIRES-RS, 435

Alcator, 16, 422

Alcator C-Mod, 10, 217, 350, 355, 483

Alfvén and Drift Waves in Plasmas, 29

Analogs with Geophysical Fluids, 184

Analytical TEM-ITG Drift-Wave Model, 320

Anisotropy-driven growth rates, 275

anomalous transport, 278

ASDEX, 10, 339, 444

ASDEX Upgrade, 225

ATC, 16

\section{BALDUR, 134}

Ballooning Interchange Modes and the

Trapped Particle Instability, 144

Basic Concepts and Historical

Background, 1

Basic Laboratory Experiments for Drift

Waves with Uniform Temperature

Profiles, 122

CGP, 490

CLM, 483

Closure Models for Coupled Chain of

Fluid Moments, 100

Collisional Transport Equations, 94

Columbia Linear Machine, 88

Compact High-Field Tokamaks, 420

Confinement Records, 14

Controlled Nuclear Fusion, 6

Coulomb collisional resistivity, 125

CRAY XT5, 484

CRONOS, 134, 343, 363

cross-field beam plasma, 274
CTEM, 435, 486

current ramp discharges, 432

Current, Density and Temperature

Gradient Driven Drift Modes, 98

CYCLONE, 480

D-T fusion plasmas, 17

Density gradients, 68

DIII-D, 355, 488

Discovering the Trapped Particle

Instability, 146

Dispersion relation, 272

Double Adiabatic Pressure Tensor for Anisotropically Heated Plasmas, 140

Double Tearing Mode, 393

Doublet III, 10

Drift Wave Eigenmodes, 45

Drift wave eigenmodes, 71

Drift Wave Eigenmodes with Active

Impurity Components, 204

Drift Wave Mechanism, 67

Drift Wave Models for LAPD, 232

Drift Wave Paradigms: Hasegawa-Mima and Hasegawa-Wakatani Models, 86

Drift Wave Turbulence, 65, 478

Drift Waves, 42

Drift Waves and Transport, 61

Drift Waves in Early Q-Machine Experiments, 125

Drift waves in the ballooning representation, 299

Dynamic behavior of ITBs, 470

EAST, 23

ECRH, 352

ECRH Driven Discharges, 352 
Effects of Drift Wave Turbulence on Magnetic Reconnection, 385

EFIT, 484, 485

electromagnetic fluctuations, 277

Electromagnetic Vortices, 248

Electron Diffusion from Magnetic Flutter in Tokamaks, 277

electron response functions, 321

Electron scale magnetic reconnection model, 408

Electron Scale Reconnection, 406

Electron Temperature Gradient Transport, 345

Electron Temperature Gradient Turbulence Modeling, 356

Electron thermal transport, 283

Electron Thermal Transport Barriers in Tokamaks, 466

Electron Thermal Transport in TCV, 349

Electron Transport and the Critical Temperature Gradient, 338

Electron viscosity, 392

ELM-free H-modes, 135, 136

ELMs, 19, 22, 108, 349, 350, 354-356, 386, 390, 483

ELMy H-mode, 135, 386

EMICs, 275

Energetic Particle Transport, 488

Energy Bounds for Turbulence Amplitudes, 68

EP-modes, 304

Equilibrium, 392

ETG, 8, 69, 108, 259, 320, 339, 340, 414, $427,489,490$

Experimental Discovery of the Trapped Particle Instabilities, 145

Experimental Measurement of Vorticity Dynamics Studies and the Reynolds Stress, 245

Fast Wave Ion Cyclotron, 343

Field Reversed Configuration, 444

FIRE, 423, 435

First generation tokamaks, 16

Fluctuating Electromagnetic Fields, 267

Fluctuating Magnetic Fields, 266

Fluctuations in the ballooning mode representation, 298

Formation of magnetic islands, 389

Franklin, 483
Frascati tokamak, 217

Frascati Tokamak Upgrade, 217, 339

fusion reactivity, 4

FWICRH, 343

GAMS, 180

GEM, 479, 490

GEM code, 490

GENE, 479

geoacoustic modes, 180

geodesic mode, 193

GKV, 479, 483

GKV-X, 296

Grad-Shafranov equilibria, 445

gravity, 14

GS2, 479, 483, 490

GT3D, 479

GT5D, 479

GTC, 479, 484, 486

GTC-P, 486

GTS, 479, 486

GYRO, 479, 483, 490

Gyro-Averaged, 24

Gyrokinetic and Computer Simulations, 478

Gyrokinetic Toroidal Code, 484

H-mode, 18, 76, 135, 173, 205, 240, 294, 349, 406, 426, 430, 444, 461, 481

Helical Toroidal Systems: LHD, Heliotrons, and Stellarators, 293

Helimak Confinement Experiment with Controlled Plasma Flows, 153

Helimak geometry, 153

HIPB, 356

hyper-resistivity, 392

I-modes, 205, 341, 430

ICRH, 22, 432

Ignition Experiments, 427

Ignition Projections, 420

IGNITOR, 437

Improved confinement modes, 453

Impurity Transport in High-Density Regimes, 217

Inertial Spectral Ranges in 2D and 3D

Turbulence, 196

Interchange stability, 137

Internal Transport Barrier Formation, 461

Internal Transport Barriers, 467 
Internal Transport Barriers for ITG/TEM Models, 325

Introduction, 376

Ion Acoustic Wave Turbulence, 51

Ion acoustic waves, 99

Ion Temperature Gradient Induced

Transport, 192

Ion Temperature Gradient Instabilities, 308

Ion temperature gradient instability, 99

Ion Temperature Gradient Mode, 82

ISX-B, 10

ITER, 6, 8-11, 18, 19, 22-24, 67, 108, 135, 216, 225, 421, 422, 428, 432, 435, 439

ITER-FEAT, 423

ITER89-P, 428

ITERH-93P, 19

ITERH-EPS, 19

ITG, 8, 69, 101, 173, 192, 300, 317, 339, $391,427,464,480,484,490$

ITG-TEM, 340

JAERI, 15, 18

JET, 14, 15, 18, 22, 23, 108, 202, 225, 227, 228

JETTO, 434, 437

JOREK, 355

JT60, 10

JT60-U, 15, 18, 108, 109, 135, 308, 327

JT60SA, 24, 491

JT60SuperAdvanced, 24

JT60U, 108

Kelvin-Helmholtz Instability and Vortices in Magnetized Plasma, 230

Kinetic Alfvén Waves, 42

Kinetic Ballooning Mode Instability, 114

Kinetic Dispersion Relation, 39

Kinetic theory, 286

Kinetic Theory Formulas, 267

KSTAR, 23

Kurchatov Nuclear Laboratory, 15

L-mode, 23, 54, 135, 294, 426, 444, 462, 479

L-modes, 341

Laboratory Experiments for Drift Waves, 119

Laboratory Plasma Drift Waves in Cylinders, 152
LAPD, 237, 483

Large Helical System Fields and

Transport, 298

Lawson condition, 6

LHCD, 22

LHCD Driven Discharges and Anisotropic Electron Phase-Space Distribution

Functions, 362

linear dynamics, 162

Linear eigenmodes, 393

LOMs, 479

Low Frequency Wave Dispersion

Relations, 29

Magnetic Confinement Conditions, 6

magnetic fluctuations, 283

Magnetic flutter thermal transport, 113

Magnetic islands, 387

Magnetic Reconnection Instabilities, 405 magnetic shear, 77

Mass Flows and Transport of Impurities in the Tokamak, 225

MAST, 339

Maxwellian distribution, 272

MHD Equations, 392

MHD equations, 129

MHD stable plasmas, 134

MHD-Magnetohydrodynamic Model, 128

mirror machines and cylindrical plasmas, 448

Mixing of the eigenmode parities, 390

MMM, 434

MMM2000, 438

MMM95, 437

MMM95 Transport Codes, 434

Momentum Stress Tensor Stability

Analysis, 114

Momentum Turbulent Transport, 488

Multi-Mode Models, 464

multiple mode transport model (MMM), 435

Multiscale Codes, 461

Multiscale Plasma Turbulence, 420

NBI, 22

NCM, 103

Neoclassical Dynamics and Transport in Toroidal Systems, 296

Nonlinear Drift Wave Models, 188 
Nonlinear dynamics of the sawtooth events, 380

Nonlinear Three-Mode Interactions and Drift-Wave Turbulence in a Tokamak Edge Plasma, 193

nonperturbative (full- $f$ ), 485

NSTX, 339, 491

Nuclear Fusion Power Reactors, 490

Numerical Eigenmodes of Resistive Viscous Tearing Modes, 403

Ohm's law, 113

ONETWO, 484, 485

ORB5, 479

ORMAK, 16

Ormak, 16

Outlook for Future of Toroidal Magnetic

Confinement Systems, 490

P-mode, 429

particle dynamics, 148

PBX-M, 10

PDX, 10, 18

Perturbative $(\delta f), 485$

phase shift, 8

Physical constants, 15

Plasma energy, 138

Plasma Turbulence, 7

PLT, 16

Polarization relations, 277

Predictive Transport Simulations, 467

Pressure Gradient Driven Instabilities, 104

Quasilinear Fluctuation, 276

quasilinear fluxes, 206

radioactivity, 227

Ranger, 483

Rayleigh-Taylor (also in Fig. 1.5), 11

Rayleigh-Taylor instability, 450

resistivity and electron viscosity, 397

reversed magnetic shear, 432

reversed shear plasmas, 487

RI-mode, 430

Rotating Magnetic Island, 414

Rotation in Cylinders, 443

Rotation in Cylindrical Plasma, 446

Rotation in tokamaks: transport and stability, 452

Rotational Instabilities, 443
S-mode, 429

Scale invariance properties, 110

Scandium and Titanium Transport, 223

SDO, 3

Self-Consistent Fields, 271

Shear Alfvén wave stabilization, 450

Sheared Magnetic Field, 45

SI, 35

slab model, 160

SOHO, 3

Solid body rotation eigenmodes, 447

Space and Astrophysics, 2

Stable double kink modes, 397

STEREO, 3

TAE-modes, 304

Tandem Mirror central cells, 444

Taroni-Bohm Thermal Transport Coefficient, 433

TCV, 339, 352

TEM, 87, 323, 435, 464, 490

TEM-ITG, 206

TEXT, 220

TEXT Tokamak, 61

TEXT tokamak, 356

TEXTOR, 10

TFR, 16

TFTR, 15, 17, 432

TFTR machine, 74

The Brotherhood of the Bomb, 5

Thermalization of Impurities and Collisional Fluxes, 221

Third-generation tokamaks, 17

Three-Component Fluid Equations, 210

TMX, 451

TMX experiment, 450

Tokamak Confinement, 13

Tokamak System with High Temperature Plasmas, 292

Tore Supra, 339

Toroidal Alfvén Eigenmodes, 301

Toroidal Confinement Systems, 292

toroidal octupole, 123

Toroidal Octupoles and Field Reversed

Configurations, 169

toroidal plasma dynamics, 177

TRACE, 3

Trace Impurity Transport Studies in TEXT, 217

TRANSP, 134, 484, 485 
Transport Mechanism in ETG

Turbulence, 489

Transport Simulations, 461

Trapped electron mode (inside the SOL), 213

Turbulent Impulsive Magnetic Energy

Release, 406

Turbulent Transport, 427

Two-Space-Scale Analysis and Dispersion

Relation, 396

Validation Analysis of the Electron

Transport Modeling, 361

vortex, $8,230,247$

vortex boundary value problems, 249

Vorticity probe measurements, 241
WAVE, 343

Wave Boundary Conditions, 48

Wave energies, 452

Wave-Particle Decorrelation, 489

Weak Turbulence Theory for Drift Waves, 79

Weiland ITG/TEM Transport Model, 330

WINDMI, 479

World War II, 5

XGC0, 481 